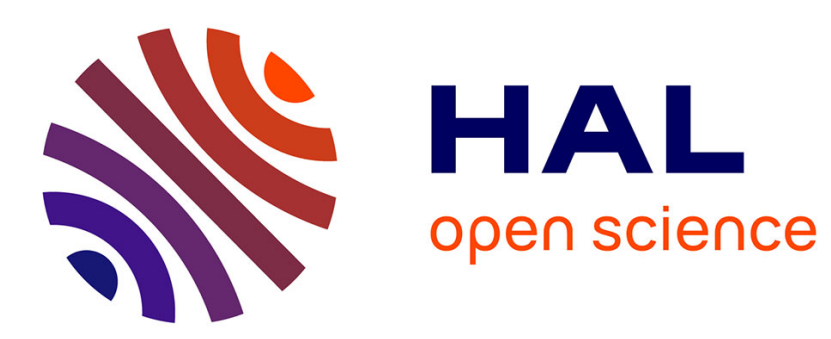

\title{
A Separation Principle for Underactuated Lossless Lagrangian Systems
}

Sofía Avila-Becerril, Antonio Loria, Elena Panteley

\section{To cite this version:}

Sofía Avila-Becerril, Antonio Loria, Elena Panteley. A Separation Principle for Underactuated Lossless Lagrangian Systems. IEEE Transactions on Automatic Control, 2017, 62 (10), pp.5318-5323. 10.1109/TAC.2016.2633782 . hal-01744620

\section{HAL Id: hal-01744620 \\ https://hal.science/hal-01744620}

Submitted on 5 Mar 2020

HAL is a multi-disciplinary open access archive for the deposit and dissemination of scientific research documents, whether they are published or not. The documents may come from teaching and research institutions in France or abroad, or from public or private research centers.
L'archive ouverte pluridisciplinaire HAL, est destinée au dépôt et à la diffusion de documents scientifiques de niveau recherche, publiés ou non, émanant des établissements d'enseignement et de recherche français ou étrangers, des laboratoires publics ou privés. 


\title{
A Separation Principle for Underactuated Lossless Lagrangian Systems
}

\author{
Sofia Avila-Becerril ${ }^{1} \quad$ Antonio Loría $^{2}$ Elena Panteley
}

\begin{abstract}
We study under-actuated Lagrangian systems without dissipative forces, augmented by a chain of integrators. For such systems, we solve the open problem of global tracking control via position measurements only; strictly speaking, we establish uniform global asymptotic stability for the closed loop system. As a corollary, we obtain an original statement for flexible-joint robots, which closes a long-standing open problem of output feedback nonlinear control.
\end{abstract}

\section{INTRODUCTION}

Ever since the early works [1], [2] on global asymptotic (state-feedback) stabilization of robot manipulators around a time-varying trajectory, establishing such result under the assumption that only generalized positions are measured has called the attention of many researchers. One of the first results on this line was reported in [3] where it is proposed a nonlinear observer-based controller that ensures local asymptotic stability. Succeedingly, a large number of articles were published in which various stability properties were established, such as semiglobal asymptotic stability, e.g. in [4], [5] or global asymptotic stability in part of the coordinates, e.g., in [6]. Global asymptotic stability is particularly difficult to prove; this was done for some case-studies, such as generic onedegree-of-freedom systems [7] and particular two-degrees of freedom systems [8], [9], but also for systems with dissipative forces (such as friction) [6], [10].

Lossless systems are, by definition, systems that do not posses natural dissipative forces such as friction hence, they constitute a fairly wide class. It is only in [11], [12], [13] that the long-standing open problem of establishing uniform global asymptotic stability for the closed-loop system, was fully solved. In [11] this was established for the controller independently proposed earlier in [5], [4] while in [12], [13] global exponential stability is established using a nonlinear observer and a coordinate transformation.

For underactuated systems, the output-feedback tracking control problem is far more complex since the relative degree with respect to link positions is augmented proportionally to the number of added integrators. Even for the meaningful case of flexible-joint robots (two added integrators, hence relative degree 4) the problem remains open. In [14] the authors presented a result based on a semi-global nonlinear observer

This work was realized while the first author was visiting the other two, under sponsorship of the grant ECOS Nord M02014. The work of E. Panteley is supported by the Government of Russian Federation (grant 074-U01).

${ }^{1} \mathrm{~S}$. Avila-Becerril is with Facultad de Ingeniería, UNAM, Mexico. soavbec@gmail.com

${ }^{2}$ A. Loría and E. Panteley are with the CNRS. LSS-CentraleSupelec, 3 Rue Joliot Curie, 91192, France. loriallss.supelec.fr, panteley@lss.supelec.fr. E. Panteley is also with ITMO University, St. Petersbourg, Russia. for the unmeasured variables, which needs only the link positions. In [15] it is proposed a robust output-feedback link position tracking controller that ensures semi-global uniformly ultimately bounded link position tracking. In the same spirit, based on a set of filters, in [16] the need of measuring link and actuator velocities is removed, yet at the expense of the restrictive assumption that the system is internally damped by viscous friction. In [17] a nonlinear observer-based certaintyequivalence tracking controller for Euler Lagrange systems is presented; global properties are obtained under provided that the controller can be bounded by an affine function in the position times a polynomial of the velocities.

In [18] is presented an output-feedback control method that applies to Lagrangian systems augmented by a chain of integrators (see Eq. (1) below) hence, underactuated systems. The controller is constructed following a modified backstepping design in which unavailable derivatives are replaced by approximate differentiation filters. Yet, it is assumed that each integrator variable is measured. In [19] we presented a controller for flexible-joint robots which ensures uniform global asymptotic stability for the closed-loop system under the assumption that joint, but not link velocities, are measured.

In this paper we extend the results of [18], [19] by assuming that the integrators' variables are not measured. We establish a separation principle for the same class of systems and we prove uniform global asymptotic stability of the origin. A significant corollary of our main result is a statement for the particular case of flexible-joint robots, as modelled in [20], [21]. Our controller stabilizes these systems globally using link and motor positions only. As far as we know, this is the first article where results of this nature are reported, even for the particular case of flexible-joint robots. See also the preliminary version of this paper, [22].

The rest of the paper is organized as follows. In the following section we formulate the problem under study and in Section III we present our main results. In Section IV we illustrate our findings with the case-study of flexible-joint robots, before concluding with some remarks in Section V.

\section{PROBLEM FORMULATION AND PRELIMINARIES}

Let us consider Euler Lagrange systems in which the control input enters through a chain of $m$ integrators, i.e.,

$$
\begin{aligned}
D(q) \ddot{q}+C(q, \dot{q}) \dot{q}+g(q) & =\xi_{1} \\
\dot{\xi}_{1} & =\xi_{2} \\
\vdots & \\
\dot{\xi}_{m-1} & =\xi_{m} \\
\dot{\xi}_{m} & =u
\end{aligned}
$$


where $u, \xi_{i} \in \mathbb{R}^{n}$ for all $i \leq m, m \geq 1$. The matrix $D(q)=D^{\top}(q)>0$ corresponds to the inertia matrix, $C(q, \dot{q}) \dot{q}$ contains the terms due to the Coriolis and centrifugal forces, $g$ represents the gravitational forces vectors. Then, we make the following hypothesis on the model (1).

\section{Assumption 1:}

1) The inertia matrix $D(q)$ is positive definite and uniformly bounded; more precisely, there exist positive real numbers $d_{m}$ and $d_{M}$ such that

$$
d_{m} \leq\left|D\left(q_{1}\right)\right| \leq d_{M} \quad \forall q \in \mathbb{R}^{n} .
$$

2) The matrix $C(w, y)$ is bounded in $w$ and linear in $y$ and, for each $i \leq n, \frac{\partial C}{\partial w_{i}}$ is bounded in $w$.

3) The matrix $\dot{D}(q)-2 C(q, \dot{q})$ is skew symmetric for all $q \in \mathbb{R}^{n}$.

4) There exists $k_{v}$ such that function that

$$
\exists k_{v}>0:\left|\frac{\partial g}{\partial q}\right| \leq k_{v}
$$

All the conditions above are commonly assumed to hold in related literature, especially on robot manipulators. For instance, the boundedness assumption on the inertia matrix holds for (but it is not limited to) robot manipulators composed of revolute joints only or prismatic joints only. See [23] for a complete characterization. Nonetheless, it is important to mention that the main result in [12], [24] which, however, holds only for fully-actuated systems $\left(m=0, \xi_{1}:=u\right)$, does not rely on uniform boundedness of $D$.

Furthermore, because $C$ is intrinsically related to $\dot{D}$ (see item 3), item 2) implicitly implies that $D$ has bounded partial derivatives. In turn, a consequence of item 2) is that there exists a saturation function sat: $\mathbb{R} \rightarrow \mathbb{R}$ and $k_{c}>0$ such that, for all $x, y, z \in \mathbb{R}^{n}$,

$$
|C(x, y)-C(z, y)| \leq k_{c} \operatorname{sat}(|x-z|)|y|,
$$

where $\operatorname{sat}(s):=\operatorname{sgn}(s) \min \{1,|s|\}$. Moreover, $C(x, y) z=$ $C(x, z) y$.

Under these conditions we solve the following tracking control problem: Let $t \mapsto q_{d}$ denote a given reference trajectory. Assume that it is sufficiently smooth and its first $m+2$ derivatives are uniformly bounded, i.e., there exists $k_{\delta}>0$ such that

$$
\max _{i \leq m+2}\left\{\sup _{t \geq 0}\left|q_{d}(t)\right|, \sup _{t \geq 0}\left|q_{d}^{(i)}(t)\right|\right\} \leq k_{\delta} .
$$

It is required to design an output-feedback dynamic controller

$$
\begin{aligned}
u & =u\left(t, q, \xi_{1}, x_{c}\right) \\
\dot{x}_{c} & =f\left(t, q, \xi_{1}, x_{c}\right)
\end{aligned}
$$

(that is, $x_{c} \in \mathbb{R}^{m}$ corresponds to the state of the controller) such that the origin of the closed-loop system is uniformly globally asymptotically stable.

Example 1 (Flexible-joint robots): According to [20], [21] joint flexibility in robot manipulators may be modelled using linear torsional springs; hence, if we denote by $K$ the joint stiffness ( $K=\infty$ implying no elasticity) the Lagrangian model is given by the equations:

$$
\begin{aligned}
D\left(q_{\ell}\right) \ddot{q}_{\ell}+C\left(q_{\ell}, \dot{q}_{\ell}\right) \dot{q}_{\ell}+\mathrm{g}\left(q_{\ell}\right) & =K\left(q_{a}-q_{\ell}\right) \\
J \ddot{q}_{a}+K\left(q_{a}-q_{\ell}\right) & =\tau
\end{aligned}
$$

where $\left(q_{\ell}, \dot{q}_{\ell}\right) \in \mathbb{R}^{2 n}$ denote the unactuated coordinates i.e., related to the links, while $\left(q_{a}, \dot{q}_{a}\right) \in \mathbb{R}^{2 n}$ correspond to the actuators' coordinates, and $\tau \in \mathbb{R}^{n}$ is the vector of input torques. The model (6) may be transformed into (1) with $m=2$, by defining: $q:=q_{\ell}, g(q):=\mathrm{g}\left(q_{\ell}\right)+K q_{\ell}, \xi_{1}:=K q_{a}$, $\xi_{2}:=K \dot{q}_{a}$ and applying the preliminary feedback

$$
\tau=\xi_{1}-K q_{\ell}+J K^{-1} u .
$$

To the best of our knowledge, the problem of ensuring uniform globally asymptotic stability for robot manipulators (6) using only link and actuator positions is completely open to date. The solution to this problem follows as a corollary to our main result. The latter is presented next; the former is presented in Section IV.

\section{MAIN RESULT: SEPARATION PRINCIPLE}

\section{A. The rationale}

In [18] is presented a dynamic output-feedback controller for (1) that guarantees uniform global asymptotic stability of the origin. The controller in the latter reference, however, is not of the form (5) since it depends also on the output $y^{\top}:=$ $\left[q^{\top} \xi^{\top}\right]$; for the case of flexible-joint robots, this is tantamount to assuming that the actuator's velocities are measurable. Here, we assume that the measurable output is $y^{\top}:=\left[q^{\top} \xi_{1}^{\top}\right]$, that is, no velocity is assumed to be measurable. This being said, our control design still builds upon the method described in [18] and, to avoid the measurement of $\xi_{i}$ for all $i>1$, we add a Luenberger observer. The simplicity of our control design leads naturally to a separation principle.

The control design method follows the rationale of classical backstepping control. First, we consider $\xi_{1}$ as a virtual control input to (1a) then, introducing the positive constants $k_{p_{0}}, k_{d_{0}}$, $a_{0}$, and $b_{0}$, we define the virtual control law $-c f$. [4], [5]

$$
\xi_{1}^{\star}=-k_{p_{0}} \tilde{q}-k_{d_{0}} \vartheta_{0}+D(q) \ddot{q}_{d}+C\left(q, \dot{q}_{d}\right) \dot{q}_{d}+g(q)
$$

where $\vartheta_{0}$ corresponds to the dirty derivative of $\tilde{q}$, that is,

$$
\begin{aligned}
\dot{q}_{c 0} & =-a_{0} \vartheta_{0} \\
\vartheta_{0} & =q_{c 0}+b_{0} \tilde{q} .
\end{aligned}
$$

If $\xi_{1}=\xi_{1}^{*}$, the null solution of the closed-loop system (1a), (8)-(9) is uniformly globally asymptotically stable [18, Theorem 4]. Then, to steer $\xi_{1} \rightarrow \xi_{1}^{*}$ we regard $\xi_{2}$ as a virtual tracking control input for the integrator (1b) with reference $\xi_{1}^{*}$. Proceeding recursively for each $i>2$, the variable $\xi_{i+1}$ is considered as a virtual control input to the $i$ th equation, and as a tracking reference in the $(i+1)$ th equation. After [18], we define

$$
\xi_{i+1}^{\star}=-k_{p_{i}} \tilde{\xi}_{i}+k_{d_{i}} \vartheta_{i}+\dot{\xi}_{i \circ}^{\star}, \quad \forall i \in[1, m-1]
$$




$$
\left.\begin{array}{rl}
\vartheta_{i} & =q_{c i}+b_{i} \xi_{i}^{\star}+\zeta_{i} \\
\dot{q}_{c i} & =-a_{i} \vartheta_{i} \\
\dot{\zeta}_{i} & =-\left(k_{d_{i}}-\sigma_{i}\right) \tilde{\xi}_{i}-b_{i} \dot{\xi}_{i \circ}^{\star}
\end{array}\right\} \forall i
$$

where $k_{p i}, k_{d i}, a_{i}, b_{i}$ are positive control parameters, $\sigma_{1}:=0$ and $\sigma_{i}:=b_{i} k_{p_{i-1}}$ for all $i \in[2, m]$.

The terms $\tilde{\xi}_{i}:=\xi_{i}-\xi_{i}^{\star}$ correspond to the "tracking errors" for each of the integrators in (1). Note that $\dot{\xi}_{i}^{\star}$ is, in general, a function of time; this is in view of its dependence on the reference trajectory $q_{d}(t)$ and the $(i+2)$ derivatives of the latter. It is, in addition, a function of the states, including nonmeasurable variables such as $\dot{\tilde{q}}$ and its $(i-1)$ derivatives; this is due to the recursive definition of $\xi_{i}^{\star}$. The term $\dot{\xi}_{i \circ}^{\star}$ stands for the value of $\dot{\xi}_{i}^{\star}$ evaluated at the origin, that is, at $(\tilde{q}, \dot{\tilde{q}}, \vartheta, \tilde{\xi})=$ $(0,0,0,0)$. In other words, $\dot{\xi}_{i \circ}^{\star}$ depends only on $t$, through $q_{d}(t)$ and the $(i+2)$ derivatives of the latter. Moreover, it verifies $\dot{\xi}_{i \circ}^{\star}:=\xi_{i+10}^{\star}$-see [18] for details.

Each group of equations (10b) corresponds to the implementation of (a modified) dirty derivative of $\xi_{i}^{\star}$ which may be computed using only known or measured variables. Indeed, in the frequency domain, $(10 \mathrm{~b})$ corresponds to

$$
\vartheta_{i}=\frac{b_{i} s}{s+a_{i}} \psi_{i} \quad \Leftrightarrow \quad \vartheta_{i}=\frac{b_{i}}{s+a_{i}} \dot{\psi}_{i}
$$

where $\psi_{i}=\xi_{i}^{\star}+\zeta_{i} / b_{i}$ and $\psi_{0}=\tilde{q}$ and $s \in \mathbb{C}$ is the Laplace variable. The second representation, in terms of a low-pass filter with input $\dot{\psi}_{i}$, is useful for the purpose of analysis since it defines output-strictly passive map $\vartheta_{i} \mapsto \dot{\psi}_{i}$. In the time domain, it corresponds to

$$
\dot{\vartheta}_{i}=-a_{i} \vartheta_{i}+b_{i} \dot{\xi}_{i}^{\star}-\left(k_{d_{i}}-\sigma_{i}\right) \tilde{\xi}_{i}-b_{i} \dot{\xi}_{i \circ}^{\star}
$$

which has an equilibrium at the origin $\{z=0\}$.

Let us stress that Equation (10b) does not define an observer since $\vartheta_{i} \not \rightarrow \dot{\tilde{q}}_{i}$ however, the quotient $\vartheta_{i} / \dot{\tilde{q}}_{i}$ remains bounded and separated from zero. Hence, one may say that, asymptotically, $\vartheta_{i} \approx \dot{\tilde{q}}_{i}$ which implies that, for the purpose of global stabilization, the necessary damping may be injected through a finite-gain low-pass filter. In other words, instead of exploiting the convergence properties of solutions estimates, as it is commonly done in observer-based designs, we use the input-output properties of the stable proper filter in (11). See [18] for formal proof of this filter property. Here, it is established that for the closed-loop system (1), (8)-(10), the origin $\{z=0\}$, with

$$
z=\left[\begin{array}{lllllllll}
\tilde{q}^{\top} & \dot{\tilde{q}}^{\top} & \vartheta_{0}^{\top} & \tilde{\xi}_{1}^{\top} & \ldots & \tilde{\xi}_{m}^{\top} & \vartheta_{1}^{\top} & \cdots & \vartheta_{m}^{\top}
\end{array}\right]^{\top},
$$

is uniformly globally asymptotically stable.

\section{B. Certainty-equivalence control}

The obvious disadvantage of the controller (10) is that it relies on the measurement of $\xi_{i}$ for all $i \leq m$-see [19]. For instance, in the case of flexible-joint robots, this includes motor velocities. In order to relax this assumption we introduce a simple reduced-order Luenberger observer for the linear $\xi$ dynamics and we use a certainty-equivalence controller which includes dirty derivatives of $q$ and $\xi^{\star}$.
The linear Luenberger observer, based on the measurement of $\xi_{1}$, is defined by

$$
\begin{aligned}
\dot{\hat{\xi}}_{i} & =\hat{\xi}_{i+1}+\lambda_{i}\left(\xi_{1}-\hat{\xi}_{1}\right), \quad \forall i \in[1, m-1] \\
\dot{\hat{\xi}}_{m} & =u+\lambda_{m}\left(\xi_{1}-\hat{\xi}_{1}\right)
\end{aligned}
$$

with constant gains $\lambda_{i}>0$ for all $i \leq m$, chosen so as to render the following matrix Hurwitz:

$$
L:=\left[\begin{array}{cccc}
-\lambda_{1} & 1 & \cdots & 0 \\
\vdots & \vdots & \ddots & \vdots \\
-\lambda_{m-1} & 0 & \cdots & 1 \\
-\lambda_{m} & 0 & \cdots & 0
\end{array}\right] \otimes I_{n}
$$

Then, defining $\bar{\xi}_{i}=\hat{\xi}_{i}-\xi_{i}^{\star}$, we introduce the certainty equivalent controller

$$
\left.\begin{array}{rl}
\xi_{i+1}^{\star} & =-k_{p_{i}} \bar{\xi}_{i}+k_{d_{i}} \vartheta_{i}+\dot{\xi}_{i \circ}^{\star}, \quad \forall i \in[1, m-1] \\
\vartheta_{i} & =q_{c i}+b_{i} \xi_{i}^{\star}+\zeta_{i} \\
\dot{q}_{c i} & =-a_{i} \vartheta_{i} \\
\dot{\zeta}_{i} & =-\left(k_{d_{i}}-\sigma_{i}\right) \bar{\xi}_{i}-b_{i} \dot{\xi}_{i \circ}^{\star}
\end{array}\right\} \forall i \in[1, m]
$$

which corresponds to (10) where $\xi_{i}$ has been replaced by its estimate $\hat{\xi}_{i}$. For a detailed (non-recursive) definition of $\xi_{i}^{*}$ see [25].

Proposition 1 (Main result): Let the reference $q_{d}$ satisfy (4). Consider the system (1) under Assumption 1 and in closed loop with the controller defined by Equations (8), (9) and (14). Then, the origin of the closed-loop system is uniformly globally asymptotically stable for sufficiently large control gains. Moreover, this holds for any observer gains $\lambda_{i}$ which render $L$ in (13) Hurwitz, independently of the control gains.

The proof is constructive; we derive explicit conditions on the control gains that imply uniform global asymptotic stability.

\section{Proof of Proposition 1}

The proof relies on recognizing that the closed-loop system has a cascaded structure,

$$
\begin{aligned}
& \dot{x}_{1}=f_{1}\left(t, x_{1}\right)+g(t, x) x_{2} \\
& \dot{x}_{2}=f_{2}\left(t, x_{2}\right)
\end{aligned}
$$

where $x_{1} \in \mathbb{R}^{p}, x_{2} \in \mathbb{R}^{q}$, and the functions $f_{1}, f_{2}$, and $g$ are continuous and locally Lipschitz in $x$, uniformly in $t$, and $f_{1}$ is continuously differentiable. After [26, Lemma 2], for (15), the origin $\left(x_{1}, x_{2}\right)=(0,0)$ is uniformly globally asymptotically stable if so are the respective origins of $\dot{x}_{1}=f_{1}\left(t, x_{1}\right)$ and (15b), and the solutions of (15a) are uniformly globally bounded.

To invoke [26, Lemma 2], we first show that the closed-loop equations have the form (15) with $x_{1}^{\top}:=z$ and $x_{2}:=\xi-\hat{\xi}$ - see Section III-C.1 below. That is, the nominal system $\dot{x}_{1}=f_{1}\left(t, x_{1}\right)$ represents the tracking-errors dynamics under the measurement of $[q, \xi]$, the equation $(15 \mathrm{~b})$ defines the state estimation errors, and $g(t, x) x_{2}$ represents the errors 
generated by the use of the certainty-equivalence controller. Uniform global asymptotic stability for $\dot{x}_{1}=f_{1}\left(t, x_{1}\right)$ is established by [18, Theorem 6]. Uniformly global exponential stability for (15b) follows since $f_{2}\left(t, x_{2}\right):=L x_{2}$ and $L$ is Hurwitz by design. In addition, $g$ is actually constant since we use a reduced-order observer. Notably, this guarantees that there is no peaking [27] and that the solutions are uniformly globally bounded. The formal proof of the latter is provided in Proposition 2 (Paragraph III-C.2), following the proof-lines of [18, Theorems 5, 6].

1) The closed-loop equations: The first error equation is obtained using the identity $\xi_{1}=\tilde{\xi}_{1}+\xi_{1}^{\star}$, by replacing $\xi_{1}^{\star}$ from (8) in (1a) and adding $-C\left(q, \dot{q}_{d}\right) \dot{q}+C(q, \dot{q}) \dot{q}_{d}=0$ to the right-hand side of the latter. Then, we differentiate on both sides of (9b) and we use (9a) to obtain

$$
\begin{aligned}
& D(q) \ddot{\tilde{q}}+\left[C(q, \dot{q})+C\left(q, \dot{q}_{d}\right)\right] \dot{\tilde{q}}+k_{p_{0}} \tilde{q}+k_{d_{0}} \vartheta_{0}=\tilde{\xi}_{1} \\
& \dot{\vartheta}_{0}=-a_{0} \vartheta_{0}+b_{0} \dot{\tilde{q}} .
\end{aligned}
$$

On the other hand, Equations (1b)-(1d) are equivalent to

$$
\begin{aligned}
\dot{\tilde{\xi}}_{i} & =\xi_{i+1}^{\star}-\dot{\xi}_{i}^{\star}+\tilde{\xi}_{i+1}, \quad i \in[1, m-1] \\
\dot{\tilde{\xi}}_{m} & =u-\dot{\xi}_{m}^{\star}
\end{aligned}
$$

so, defining $e_{i}:=\xi_{i}-\hat{\xi}_{i}$ and using the identity $\bar{\xi}_{i}=\tilde{\xi}_{i}-e_{i}$ with $i \leq m$, as well as (14), we obtain

$$
\begin{aligned}
& \dot{\tilde{\xi}}_{i}=-k_{p_{i}} \tilde{\xi}_{i}+k_{d_{i}} \vartheta_{i}+\tilde{\xi}_{i+1}+k_{p_{i}} e_{i}-\left(\dot{\xi}_{i}^{\star}-\dot{\xi}_{i \circ}^{\star}\right) \\
& \dot{\vartheta}_{i}=-a_{i} \vartheta_{i}-\left(k_{d i}-\sigma_{i}\right)\left(\tilde{\xi}_{i}+e_{i}\right)+b_{i}\left(\dot{\xi}_{i}^{\star}-\dot{\xi}_{i \circ}^{\star}\right) .
\end{aligned}
$$

Next, to compact the notation, we define $A:=\operatorname{diag}\left\{a_{i}\right\} \otimes$ $I_{n}, B:=\operatorname{diag}\left\{b_{i}\right\} \otimes I_{n}, K_{d}:=\operatorname{diag}\left\{k_{d_{i}}\right\} \otimes I_{n}, K_{p}:=$ $\operatorname{diag}\left\{k_{p_{i}}\right\} \otimes I_{n}, K_{d}^{\prime}:=K_{d}-\operatorname{diag}\left\{\sigma_{i}\right\} \otimes I_{n}$,

$$
\begin{aligned}
K_{p}^{\prime} & =\left[\begin{array}{ccccc}
k_{p_{1}} & -1 & 0 & \cdots & 0 \\
0 & \ddots & \ddots & & \vdots \\
\vdots & & & & -1 \\
0 & \cdots & \cdots & 0 & k_{p_{m}}
\end{array}\right] \otimes I_{n}, \\
\tilde{\xi}^{\top}= & {\left[\begin{array}{lll}
\tilde{\xi}_{1}^{\top} & \cdots & \tilde{\xi}_{m}^{\top}
\end{array}\right] \quad \dot{\xi}_{\circ}^{\star \top}=\left[\begin{array}{llll}
\dot{\xi}_{1 \circ}^{\star \top} & \cdots & \dot{\xi}_{m \circ}^{\star \top}
\end{array}\right] } \\
\vartheta^{\top}= & {\left[\begin{array}{llll}
\vartheta_{1}^{\top} & \cdots & \vartheta_{m}^{\top}
\end{array}\right] \quad e^{\top}=\left[\begin{array}{llll}
e_{1} & \cdots & e_{m}
\end{array}\right] . }
\end{aligned}
$$

Then, the tracking-error-dynamics equations (18) become

$$
\begin{aligned}
& \dot{\tilde{\xi}}=-K_{p}^{\prime} \tilde{\xi}+K_{d} \vartheta+K_{p} e-\left[\dot{\xi}^{\star}-\dot{\xi}_{\circ}^{\star}\right] \\
& \dot{\vartheta}=-A \vartheta-K_{d}^{\prime} \tilde{\xi}+K_{d}^{\prime} e+B\left[\dot{\xi}^{\star}-\dot{\xi}_{\circ}^{\star}\right]
\end{aligned}
$$

while the estimation-error-dynamics is

$$
\dot{e}=L e
$$

in which $L$ is Hurwitz by design.

Note that the equations (19) consitute a linear time-invariant system that is perturbed, on one hand, by the exponentiallyconverging input $e(t)$ and, on the other, by the "input" $\left[\dot{\xi}^{\star}-\dot{\xi}_{\circ}^{\star}\right]$ which vanishes at the origin hence, provided that $(\tilde{q}, \dot{\tilde{q}}, \vartheta) \rightarrow(0,0,0)$. To see this, we remark that long but straightforward computations ( $c f$. [25]) lead to the identity

$$
\dot{\xi}_{i}^{\star}=-k_{p_{i-1}} \tilde{\xi}_{i}+\sum_{k=1}^{i-1} \eta_{i k} \tilde{\xi}_{k}-\mu_{i k} \vartheta_{k}+k_{p_{i-1}} e_{i}-\sum_{k=2}^{i-1} \eta_{i k} e_{k}
$$

$$
-\sum_{k=1}^{i-1} \gamma_{i 1} e_{1}+\prod_{j=1}^{i-1} \beta_{j}\left[\dot{\xi}_{1}^{\star}-\dot{\xi}_{1 \circ}^{\star}\right]+\dot{\xi}_{i \circ}^{\star},
$$

where $\beta_{j}=k_{p j}+b_{j} k_{d_{j}}, j \in[1, m-1]$ and, for each $i \in$ $[2, m]$ and $k \in[1, i-1]$,

$$
\begin{aligned}
\eta_{i k} & =\prod_{j=k+1}^{i} \beta_{j}^{\operatorname{sgn}(i-j)}\left[k_{p_{k}}^{2}-k_{d_{k}}^{2}-k_{p_{k}} k_{p_{k-1}} \operatorname{sgn}(k-1)\right], \\
\mu_{i k} & =\prod_{j=k+1}^{i} \beta_{j}^{\operatorname{sgn}(i-j)} k_{d_{k}}\left[k_{p_{k}}+a_{k}\right], \\
\gamma_{i 1} & =\prod_{j=1}^{i-1} \beta_{j+1}^{\operatorname{sgn}(i-j-1)} k_{p_{j}} \lambda_{j} .
\end{aligned}
$$

Therefore,

$$
\dot{\xi}^{\star}-\dot{\xi}_{\circ}^{\star}=\Gamma_{1} \tilde{\xi}+\Gamma_{2} \vartheta-\Gamma_{4} e+\Gamma_{3}\left[\dot{\xi}_{1}^{\star}-\dot{\xi}_{10}^{\star}\right]
$$

where

$$
\begin{gathered}
\Gamma_{1}=\left[\begin{array}{cccc}
0 & 0 & \cdots & 0 \\
\eta_{21} & -k_{p_{1}} & & \vdots \\
\vdots & & \ddots & \\
\eta_{m_{1}} & \eta_{m_{2}} & \cdots & -k_{p_{m-1}}
\end{array}\right] \otimes I_{n}, \quad(22) \\
\Gamma_{2}=\left[\begin{array}{cccc}
0 & 0 & \cdots & 0 \\
-\mu_{21} & 0 & & \vdots \\
\vdots & & \ddots & \\
-\mu_{m_{1}} & -\mu_{m_{2}} & \cdots & 0
\end{array}\right] \otimes I_{n}, \quad \Gamma_{3}=\left[\begin{array}{c}
1 \\
\beta_{1} \\
\beta_{1} \\
\vdots
\end{array}\right] \otimes I_{n}, \\
\Gamma_{4}=\left[\begin{array}{cccc}
0 & 0 & \cdots & 0 \\
\eta_{21}+\gamma_{21} & -k_{p_{1}} & & \vdots \\
\vdots & & \ddots & \\
\eta_{m_{1}}+\gamma_{m_{1}} & \eta_{m_{2}} & \cdots & -k_{p_{m-1}}
\end{array}\right] \otimes I_{n} . \quad \text { (23) }
\end{gathered}
$$

Thus, replacing (21) in (19) we obtain

$$
\begin{gathered}
\dot{\tilde{\xi}}=-\left(K_{p}^{\prime}+\Gamma_{1}\right) \tilde{\xi}+\left(K_{d}-\Gamma_{2}\right) \vartheta \\
-\Gamma_{3}\left[\dot{\xi}_{1}^{\star}-\dot{\xi}_{1 \circ}^{\star}\right]+\left(K_{p}^{\prime}+\Gamma_{1}\right) e \\
\dot{\vartheta}=-\left(A-B \Gamma_{2}\right) \vartheta-\left(K_{d}^{\prime}-B \Gamma_{1}\right) \tilde{\xi} \\
\quad+B \Gamma_{3}\left[\dot{\xi}_{1}^{\star}-\dot{\xi}_{1 \circ}^{\star}\right]+\left(K_{d}^{\prime}-B \Gamma_{1}\right) e
\end{gathered}
$$

which corresponds to a stable linear system perturbed by the linear-sector non-linearity $\left[\dot{\xi}_{1}^{\star}-\dot{\xi}_{10}^{\star}\right]$. Now, Assumption 1 together with (4) guarantee that there exist non-negative reals $\eta_{1}, \eta_{2}$ and $\eta_{3}$ as well as a continuous odd saturation function sat $: \mathbb{R} \rightarrow \mathbb{R}$ such that $|\operatorname{sat}(y)| \leq 1$, and

$$
\left|\left[\dot{\xi}_{1}^{\star}-\dot{\xi}_{1 \circ}^{\star}\right]\right| \leq \eta_{1} \operatorname{sat}(|\tilde{q}|)+\eta_{2}|\dot{\tilde{q}}|+\eta_{3}\left|\vartheta_{0}\right| .
$$

Furthermore, if we define $x:=\left[\tilde{\xi}^{\top} \vartheta^{\top}\right]^{\top}$ the system (24) becomes

$$
\dot{x}=\mathcal{A} x+\mathcal{B}\left[\dot{\xi}_{1}^{\star}-\dot{\xi}_{1 \circ}^{\star}\right]+K^{\prime} e
$$

where

$\mathcal{A}=\left[\begin{array}{cc}-\left(K_{p}^{\prime}+\Gamma_{1}\right) & K_{d}-\Gamma_{2} \\ -\left(K_{d}^{\prime}-B \Gamma_{1}\right) & -\left(A-B \Gamma_{2}\right)\end{array}\right], \quad \mathcal{B}=\left[\begin{array}{c}-\Gamma_{3} \\ B \Gamma_{3}\end{array}\right]$, 


$$
K^{\prime}=\left[\begin{array}{c}
K_{p}+\Gamma_{1}^{\prime} \\
K_{d}^{\prime}-B \Gamma_{1}
\end{array}\right]
$$

Thus, the closed-loop dynamics is now in the convenient cascaded form (15) with $x_{1}=z, x_{2}:=e, f_{2}\left(t, x_{2}\right)=L e$, and $f_{1}\left(t, x_{1}\right)$ corresponds to the right-hand side of

$$
\left\{\begin{array}{l}
\text { Eq. (16) } \\
\dot{x}=\mathcal{A} x+\mathcal{B}\left[\dot{\xi}_{1}^{\star}-\dot{\xi}_{10}^{\star}\right] .
\end{array}\right.
$$

2) Boundedness of solutions:

Proposition 2: Let $L$ and $\mathcal{A}$ be Hurwitz and let

$$
k_{d_{0}}\left[\frac{a_{0}}{4 b_{0}}-m\right]>\frac{m+2}{2}+\frac{\left[2 k_{c} k_{\delta}+(m+2) / 2\right] a_{0}^{2}}{b_{0}^{2}} .
$$

Assume, also, that there exist positive definite matrices $P$ and $Q$ such that $Q=-\left(\mathcal{A}^{\top} P+P \mathcal{A}\right)$ and

$$
Q>\frac{1}{2} \operatorname{diag}\{Q\}>\left(\eta_{2}^{2}+\eta_{3}^{2}\right) \operatorname{diag}\left\{\left|[P \mathcal{B}]_{i}\right|^{2}\right\},
$$

where $[P \mathcal{B}]_{i}$ is the $i$ th $n \times n$ block of $P \mathcal{B} \in \mathbb{R}^{m n \times n}$ and $\eta_{2}$, $\eta_{3}$ are defined in (25). Then, the solutions of the closed-loop system (16), (20), (26) are uniformly globally bounded.

Proof. The solutions of (20) are uniformly globally bounded since $L$ is Hurwitz. The rest of the proof follows that of [18, Theorem 6]. Let the Hurwitz property of $L$ generate positive definite matrices $P_{L}$ and $-Q_{L}:=\left(L^{\top} P_{L}+P_{L} L\right)$ and let $\kappa>0$ be a real constant. Then, consider the function $W$ : $\mathbb{R}^{2 m n} \times \mathbb{R}^{m n} \rightarrow \mathbb{R}_{\geq 0}$, defined as

$$
W(x, e)=\kappa x^{\top} P x+e^{\top} P_{L} e ;
$$

a direct computation shows that its total derivative along the trajectories of (20), (26) yields

$\dot{W}(x)=\kappa\left[x^{\top} Q x+2 x^{\top} P \mathcal{B}\left[\dot{\xi}_{1}^{\star}-\dot{\xi}_{1 \circ}^{\star}\right]+2 x^{\top} P K^{\prime} e\right]-e^{\top} Q_{L} e$.

Next, define $c:=\left|P K^{\prime}\right|, \lambda:=\lambda_{\min }\left(Q_{L}\right)$; using the CauchySchwartz and the triangle inequalities, we obtain

$$
\begin{aligned}
\dot{W} \leq & -\kappa x^{\top} Q x+2 \kappa x^{\top} P \mathcal{B}\left[\dot{\xi}_{1}^{\star}-\dot{\xi}_{1 \circ}^{\star}\right]+\frac{\kappa c}{\epsilon}|x|^{2} \\
& -(\lambda-\kappa c \epsilon)|e|^{2}
\end{aligned}
$$

for any $\epsilon>0$. Furthermore,

$$
\begin{aligned}
& x^{\top} P \mathcal{B}\left[\dot{\xi}_{1}^{\star}-\dot{\xi}_{10}^{\star}\right]=\left(\sum_{i=1}^{m} \tilde{\xi}_{i}^{\top}[P \mathcal{B}]_{i}+\vartheta_{i}^{\top}[P \mathcal{B}]_{m+i}\right)\left[\dot{\xi}_{1}^{\star}-\dot{\xi}_{1 \circ}^{\star}\right] \\
& \leq \frac{1}{2}\left(\sum_{i=1}^{m}\left|\tilde{\xi}_{i}\right|^{2}\left|[P \mathcal{B}]_{i}\right|^{2}+\left|\vartheta_{i}\right|^{2}\left|[P \mathcal{B}]_{m+i}\right|^{2}\right)\left(\eta_{2}^{2}+\eta_{3}^{2}\right) \\
& +\eta_{1}\left(\sum_{i=1}^{m}\left|\tilde{\xi}_{i}\right|\left|[P \mathcal{B}]_{i}\right|+\left|\vartheta_{i}\right|\left|[P \mathcal{B}]_{m+i}\right|\right)+\frac{m}{2}\left[|\dot{\tilde{q}}|^{2}+\left|\vartheta_{0}\right|^{2}\right] .
\end{aligned}
$$

Therefore, in view of the second inequality in (29) we have

$$
\begin{aligned}
\dot{W} \leq & -\kappa x^{\top}\left(Q-\frac{1}{2} \operatorname{diag}\{Q\}-\frac{c}{\epsilon} I\right) x-(\lambda-\kappa c \epsilon)|e|^{2} \\
& +2 \kappa \eta_{1}\left(\sum_{i=1}^{m}\left|\tilde{\xi}_{i}\right|\left|[P \mathcal{B}]_{i}\right|+\left|\vartheta_{i}\right|\left|[P \mathcal{B}]_{m+i}\right|\right) \\
& +\kappa m\left[|\dot{\tilde{q}}|^{2}+\left|\vartheta_{0}\right|^{2}\right]
\end{aligned}
$$

Next, let $\mathcal{V}: \mathbb{R}_{\geq 0} \times \mathbb{R}^{3 n(m+1)} \rightarrow \mathbb{R}_{\geq 0}$ be defined as $V\left(t, \tilde{q}, \dot{\tilde{q}}, \vartheta_{0}\right)+W(x, e)$, where

$$
V=\frac{\kappa}{2}\left(\dot{\tilde{q}}^{\top} D\left(\tilde{q}+q_{d}(t)\right) \dot{\tilde{q}}+k_{p_{0}}|\tilde{q}|^{2}+\frac{k_{d_{0}}}{b_{0}}\left|\vartheta_{0}\right|^{2}\right)
$$

The total derivative of $\mathcal{V}:=V+W$ along the trajectories of (27) yields

$$
\begin{aligned}
& \dot{\mathcal{V}} \leq-\kappa x^{\top}\left[Q-\frac{1}{2} \operatorname{diag}\{Q\}-\frac{c}{\epsilon} I\right] x-(\lambda-\kappa c \epsilon)|e|^{2} \\
&+\frac{\kappa}{2}\left|\tilde{\xi}_{1}\right|^{2}+\kappa\left(m+2+k_{c} k_{\delta}\right)|\dot{\tilde{q}}|^{2}-\kappa\left[\frac{a_{0} k_{d_{0}}}{b_{0}}-m\right]\left|\vartheta_{0}\right|^{2} \\
& \quad+2 \kappa \eta_{1}\left(\sum_{i=1}^{m}\left|\tilde{\xi}_{i}\right|\left|[P \mathcal{B}]_{i}\right|+\left|\vartheta_{i}\right|\left|[P \mathcal{B}]_{m+i}\right|\right)
\end{aligned}
$$

The rest of the proof of boundedness follows as for Theorem 6 in [18]. The result follows from [26, Lemma 2].

Finally, to see that the separation principle holds, let the control gains be given according to (28) and (29). Let the observer gain $L$ be any given Hurwitz matrix generating $\lambda$ in (31). Then, there exists a sufficiently large $\epsilon$ such that the matrix $Q-(1 / 2) \operatorname{diag}\{Q\}-(c / \epsilon) I$ is positive definite. On the other hand, for any given $\lambda$ and $\epsilon$ there exists $\kappa$ such that the factor $(\lambda-\kappa c \epsilon)$ is positive. Therefore, the controller and the observer gains are independent of each other.

\section{CASE-STUdY: FLEXIBLE-JOINT ROBOTS}

We briefly present a corollary of Proposition 1 which, nevertheless, solves the longstanding open problem of positionfeedback global tracking control for flexible-joint manipulators, (6). Moreover, we establish a separation principle.

The problem consists in stabilizing the trajectory $t \mapsto$ $\left(q_{d}, \dot{q}_{d}, K^{-1} \xi_{1 \circ}^{\star}, K^{-1} \dot{\xi}_{1 \mathrm{o}}^{\star}\right)$ via measurement of $q$ and $\xi_{1}$ only, which is equivalent to stabilizing (1) at $\left(q_{d}, \dot{q}_{d}, \xi_{1 \circ}^{\star}, \xi_{2 \circ}^{\star}\right)$. To that end consider the reduced-order linear observer

$$
\begin{aligned}
& \dot{\hat{\xi}}_{1}=\hat{\xi}_{2}+\lambda_{1}\left(\xi_{1}-\hat{\xi}_{1}\right), \\
& \dot{\hat{\xi}}_{2}=u+\lambda_{2}\left(\xi_{1}-\hat{\xi}_{1}\right)
\end{aligned}
$$

where $\lambda_{1}$ and $\lambda_{2}$ are positive constants. In addition, defining $\bar{\xi}=\hat{\xi}-\xi^{\star}$, consider the certainty-equivalence controller

$$
\left.\begin{array}{rl}
\xi_{2}^{\star} & =-k_{p_{1}} \bar{\xi}_{1}+k_{d_{1}} \vartheta_{1}+\dot{\xi}_{10}^{\star}, \\
\vartheta_{i} & =q_{c i}+b_{i} \xi_{i}^{\star}+\zeta_{i} \\
\dot{q}_{c i} & =-a_{i} \vartheta_{i} \\
\dot{\zeta}_{i} & =-\left(k_{d_{i}}-\sigma_{i}\right) \bar{\xi}_{i}-b_{i} \dot{\xi}_{i \circ}^{\star}
\end{array}\right\} \forall i \in[1,2]
$$

where $k_{p i}$ and $k_{d i}$, which denote "proportional" and "derivative" control gains respectively, are positive constants.

Proposition 3: Let $q_{d}$ be given such that (4) holds. Consider the system (6) under Assumption 1 in closed loop with the controller defined by Equations (7)-(35). Let the controller gains be such that: $k_{p_{1}}=k_{d_{1}}$;

$$
\frac{k_{d_{0}}}{2}\left[\frac{a_{0}}{4 b_{0}}-2\right]>\frac{\left(k_{c} k_{\delta}+1\right) a_{0}^{2}}{b_{0}^{2}}+1
$$




$$
\begin{array}{r}
\min \left\{k_{p_{1}}, \frac{\left[k_{p_{2}}-k_{p_{1}}\right]}{\beta^{2}}, \frac{a_{1}}{b_{1}^{2}}, \frac{a_{2}}{b_{2}^{2} \beta^{2}}\right\}>\left[\eta_{2}^{2}+\eta_{3}^{2}\right] \\
{\left[k_{p_{2}}-k_{p_{1}}\right]>\max \left\{\frac{2}{k_{p_{1}}}, \frac{4 \mu^{2}}{a_{1}}\right\}, \quad a_{1} a_{2}>2 b_{2}^{2} \mu^{2}}
\end{array}
$$

where $\mu=k_{p 1}\left(k_{p 1}+a_{1}\right)$, and $\beta=k_{p_{1}}\left(1+b_{1}\right)$. Then, the origin of the closed-loop system is uniformly globally asymptotically stable for sufficiently large control gains and any positive observer gains $\lambda_{1}, \lambda_{2}$.

Sketch of Proof. The key observation is that since $k_{p_{1}}=k_{d_{1}}$ then $\eta_{21}:=0$ in (22) and (23) hence, the matrix $P$ in Proposition 2 may be chosen as the identity. Moreover, in view of (36) and (38) the resulting matrix

$$
Q=\left[\begin{array}{cccc}
2 k_{p_{1}} & -1 & 0 & 0 \\
-1 & 2\left[k_{p_{2}}-k_{p_{1}}\right] & -\mu & 0 \\
0 & -\mu & 2 a_{1} & -b_{2} \mu \\
0 & 0 & -b_{2} \mu & 2 a_{2}
\end{array}\right] \otimes I_{n}
$$

(defined in Proposition 2) is positive definite. Actually, (36) implies (29) while (38) implies that $Q-(1 / 2) \operatorname{diag}\{Q\}$ is positive definite. In addition, (36) corresponds to (28). Inequality (33) becomes

$$
\begin{aligned}
\dot{\mathcal{V} \leq} \leq & -\kappa x^{\top}\left(Q-\frac{1}{2} \operatorname{diag}\{Q\}-\frac{c}{\epsilon} I\right) x-(\lambda-\kappa c \epsilon)|e|^{2} \\
& +\frac{\kappa}{2}\left|\tilde{\xi}_{1}\right|^{2}+2 \kappa \eta_{1}\left(\left|\tilde{\xi}_{1}\right|+\beta\left|\tilde{\xi}_{2}\right|+b_{1}\left|\vartheta_{1}\right|+b_{2} \beta\left|\vartheta_{2}\right|\right) \\
& +\kappa\left(k_{c} k_{\delta}+4\right)|\dot{\tilde{q}}|^{2}-\kappa\left[\frac{a_{0} k_{d_{0}}}{b_{0}}-2\right]\left|\vartheta_{0}\right|^{2} .
\end{aligned}
$$

For the purpose of establishing uniform global boundedness, note that the negative quadratic terms in $|x|^{2}$ dominate over all terms involving (large) $\left|\tilde{\xi}_{i}\right|$ and $\left|\vartheta_{i}\right|$ while the sum of the last two terms in the right hand side generate a sequence of negative numbers for any increasing sequence $\left\{t_{i}\right\}$ such that $\left\{\left|\tilde{q}\left(t_{i}\right)\right|\right\}$ is also monotonically increasing -see [18] for details.

\section{CONCLUSIONS}

We have presented the first position feedback controller for flexible-joint manipulators guaranteeing uniform global asymptotic stability of the origin for the closed-loop system. This has been done by establishing a separation principle for a passivity-based controller which makes use of approximate differentiation of link positions and a reduced-order Luenberger observer for the motor velocities. The scheme enters in a general framework that applies to a wide class of underactuated Euler-Lagrange systems of arbitrary relative degree (with respect to the measured output).

\section{REFERENCES}

[1] J. J. Slotine and W. Li, "Adaptive manipulator control: a case study," IEEE Trans. on Automat. Contr., vol. AC-33, pp. 995-1003, 1988.

[2] B. Paden and R. Panja, "Globally asymptotically stable PD+ controller for robot manipulators," Int. J. of Contr., vol. 47, pp. 1697-1712, 1988.

[3] S. Nicosia and P. Tomei, "Robot control by using only joint position measurement," IEEE Trans. on Automat. Contr., vol. 35-9, pp. 1058$1061,1990$.

[4] I. V. Burkov, "Mechanical system stabilization via differential observer," in IFAC Conference on System Structure and Control, (Nantes, France), pp. 532-535, 1995.
[5] A. Loría and R. Ortega, "On tracking control of rigid and flexible joints robots," Appl. Math. and Comp. Sci., special issue on Mathematical Methods in Robotics, K. Tchon and A. Gosiewsky, eds, vol. 5, no. 2, pp. 101-113, 1995.

[6] E. Zergeroglu, D. M. Dawson, M. S. de Queiroz, and M. Krstić, "On global output feedback tracking control of robot manipulators," in Proc. 39th. IEEE Conf. Decision Contr., (Sydney, Australia), pp. 5073-5078, 2000.

[7] A. Loría, "Global tracking control of one degree of freedom EulerLagrange systems without velocity measurements," European J. of Contr., vol. 2, no. 2, 1996.

[8] Z. P. Jiang and I. Kanellakopoulos, "Global output feedback tracking for a benchmark nonlinear system," IEEE Trans. on Automat. Contr. vol. 45, no. 5, pp. 1023-1027, 2000.

[9] Z.-P. Jiang, D. J. Hill, and Y. Guo, "Stabilization and tracking via output feedback for the nonlinear benchmark system," Automatica, vol. 34, no. 7, pp. $907-915,1998$.

[10] E. V. L. Nunes and L. Hsu, "Global tracking for robot manipulators using a simple causal PD controller plus feedforward," Robotica, vol. 28, no. 1, pp. 23-34, 2010. DOI:10.1017/S0263574709005529.

[11] A. Loria, "Uniform global position feedback tracking control of mechanical systems," in Proc. IEEE American Control Conference, (Washington, D.C.), pp. 5722-5727, 2013. DOI: 10.1109/ACC.2013.6580734.

[12] J. G. Romero-Velázquez, I. Sarras, and R. Ortega, "A globally exponentially stable tracking controller for mechanical systems using position feedback," in Proc. IEEE American Control Conference, pp. 4976-4981, 2013.

[13] J. Romero, R. Ortega, and I. Sarras, "A globally exponentially stable tracking controller for mechanical systems using position feedback," Automatic Control, IEEE Transactions on, vol. 60, pp. 818-823, March 2015.

[14] S. Nicosia and P. Tomei, "A tracking controller for flexible joint robots using only link position feedback," IEEE Trans. on Automat. Contr., vol. 40, no. 5, pp. 885-890, 1995.

[15] Y. Zhu, D. Dawson, T. Burg, and J. Hu, "A cheap output feedback tracking controller with robustness: the rlfj problem," in Robotics and Automation, 1996. Proceedings., 1996 IEEE International Conference on, vol. 1, pp. 939-944 vol.1, 1996.

[16] S. Y. Lim, D. M. Dawson, J. Hu, and M. S. De Queiroz, "An adaptive link position tracking controller for rigid-link flexible-joint robots without velocity measurements," Systems, Man, and Cybernetics, Part B: Cybernetics, IEEE Transactions on, vol. 27, no. 3, pp. 412-427, 1997.

[17] Ø. N. Stamnes, O. M. Aamo, and G.-O. Kaasa, "Global output feedback tracking control of Euler-Lagrange systems," in Proc of 18th IFAC Word Congress, pp. 215-220, 2011.

[18] A. Loria, "Observers are unnecessary for output-feedback control of Lagrangian systems," IEEE Trans. Automat. Control, vol. 61, no. 4, pp. 905-920, 2016.

[19] A. Loría and S. Avila-Becerril, "Output-feedback global tracking control of robot manipulators with flexible joints," in Proc. IEEE American Control Conference, (Portland, Oregon), pp. 4032-4037, 2014. DOI: 10.1109/ACC.2014.6858900.

[20] M. Spong, "Modeling and control of elastic joint robots," ASME J. Dyn. Syst. Meas. Contr., vol. 109, pp. 310-319, 1987.

[21] I. V. Burkov and A. T. Zaremba, "Dynamics of elastic manipulators with electric drives," Izv. Akad. Nauk SSSR Mekh. Tverd. Tela, vol. 22, no. 1, pp. 57-64, 1987. Engl. transl. in Mechanics of Solids, Allerton Press.

[22] S. Avila-Becerril, A. Loría, and E. Panteley, "Global position-feedback tracking control of flexible-joint robots," in Proc. IEEE American Control Conference, 2016. To appear.

[23] F. Ghorbel, B. Srinivasan, and M. W. Spong, "On the uniform boundedness of the inertia matrix of serial robot manipulators," J. of Robotic Systems, vol. 151, pp. 17-28, 1998.

[24] J. G. Romero, Robust Energy Shaping Control of Nonlinear Systems. $\mathrm{PhD}$ thesis, Univ Paris Sud, Feb. 2013.

[25] A. Loría, "Observer-less output feedback global tracking control of lossless Lagrangian systems." e-print no. arXiv:1307.4659, June 2013. Available from http://arxiv.org/abs/1307.4659 .

[26] E. Panteley and A. Loría, "Growth rate conditions for stability of cascaded time-varying systems," Automatica, vol. 37 , no. 3, pp. 453460, 2001.

[27] H. J. Sussman and P. V. Kokotović, "The peaking phenomenon and the global stabilization of nonlinear systems," IEEE Trans. on Automat. Contr., vol. 36, no. 4, pp. 424-439, 1991. 